\title{
Correlations Between Administration of Food Supplements with Marked Antioxidant Properties and Clinical Parameters in Patients with Prostate Carcinoma
}

\author{
Torricelli Piera ${ }^{1}$, Antonelli Francesco ${ }^{2}$, Ferorelli Pasquale ${ }^{2}$, Shevchenko Anna ${ }^{3}$, Lenzi Stefano ${ }^{4}$, \\ Beninati Simone $^{2, *}$ \\ ${ }^{1}$ Department of Urology, Hospital "A. Cardarelli", Campobasso, Italy \\ ${ }^{2}$ Department of Biology, University of Tor Vergata, Rome, Italy \\ ${ }^{3}$ Department of Science, People's Friendship University of Russia, Moscow, Russia \\ ${ }^{4}$ Department of Medicine, Université Européenne Jean Monnet, Bruxelles, Belgium \\ Email address: \\ beninati@bio.uniroma2.it(B. Simone) \\ ${ }^{*}$ Corresponding author
}

\section{To cite this article:}

Torricelli Piera, Antonelli Francesco, Ferorelli Pasquale, Shevchenko Anna, Lenzi Stefano, Beninati Simone. Correlations Between Administration of Food Supplements with Marked Antioxidant Properties and Clinical Parameters in Patients with Prostate Carcinoma. International Journal of Clinical Oncology and Cancer Research. Vol. 3, No. 2, 2018, pp. 14-19. doi: 10.11648/j.ijcocr.20180302.11

Received: March 26, 2018; Accepted: April 12, 2018; Published: May 10, 2018

\begin{abstract}
Several studies indicate that high oxidative stress is associated with various degenerative diseases, including tumors. The high levels of free radicals present in many patients derive from the chronic lack of antioxidants, caused by an increasingly poor and artificial diet. The study presented in this research, conducted on 50 male volunteers, carriers of Prostatic Carcinoma (PCa) at different stages of development and under current therapy, showed that the daily intake of antioxidants contained in two food supplements, Citozym (CIZ) and Propulzym (PRZ), induces a significant increase of the ratio free/total PSA and also a reduction of various clinical parameters, correlated with PCa. This result suggests a potential slowdown in the progression of the disease. This study was planned on the basis of a recent preliminary trial, that highlighted the positive activity of CIZ in a model of benign prostatic hypertrophy. Studies are in progress to identify the components present in these food supplements affecting biochemical signals, elements that underlie the ability of PCa to progress. This does not mean that the intake of antioxidants is a cure for $\mathrm{PCa}$, but that a high undiagnosed oxidative stress, much less correct by the standard oncological approaches, contributes to the evolution of the tumor disease. In other words, current oncology focuses correctly on reducing the tumor mass, but does not intervene on the biological medium that produced it. This is why there is still a dramatic incidence of relapses.
\end{abstract}

Keywords: Food Supplements, Antioxidants, Prostate Carcinoma, Oxidative Stress

\section{Introduction}

$\mathrm{PCa}$ is recognized as one of the main medical problems facing the male population. In Europe, it is by far the most frequent neoplasia, with an annual incidence of 214 cases per 1000 men for an estimated 2.6 million new cases each year, and the second leading cause of death by neoplasm. For about 20 years with the introduction into the clinical practice of PSA (specific prostate antigen) there has indeed been a noticeable increase in the diagnosis of the neoplasm, to which, however, has corresponded only a slight increase in mortality, posing the problem of PCa clinically not significant and the risk of over-processing. Several studies indicate that high oxidative stress is associated with many degenerative diseases including tumors. To contribute heavily to the high levels of free radicals found in patients is a chronic deficiency of antioxidants caused by an increasingly poor and artificial diet. A study carried out in 2015 highlighted the daily intake of antioxidants by patients with PCa induces a significant reduction in PSA [1]. This according to the researchers indicates a real slowing down of the progression of the disease. Recently, researchers have identified some antioxidant components of plants that seem able to inhibit the movement of cancer cells and weaken their chemical signals, elements that are at the base of the 
tumor's ability to generate metastases [2]. This is not to say that intake of antioxidants is a cure for $\mathrm{PCa}$ but that a high undiagnosed oxidative stress, much less correct by standard oncological approaches, contributes to the evolution of tumor disease. In other words, current oncology focuses correctly on reducing the tumor mass but does not intervene on the biological soil that produced it. This is why there is still a dramatically high incidence of relapses.

To date, the main diagnostic tools for prostate tumor include the digito-rectal prostate (DRE) scan, trans-rectal ultrasound and PSA. The DRE must always be the first diagnostic approach and has the purpose of appreciating the shape, the consistency, the tenderness and approximately the volume of the gland, as well as the presence of suspicious nodules. Trans-rectal ultrasound mainly performs the task of highlighting areas of doubtful ultrasound and improving the accuracy of prostate biopsy, which is the only investigation that allows to make a certain diagnosis of neoplasia. The PSA is still today the most used silky marker. It is a glycoprotein produced almost exclusively by the epithelial cells of the prostate and, fundamental data, constitutes an organ marker and not of cancer; therefore an elevation of its values can also occur in the presence of other prostatic pathologies (Benign Prostatic Hyperplasia and prostatitis). It is a serine protease of the human tissue callicreine family, encoded by a gene located on chromosome 19 , which is produced by secretory columnar epithelial cells and is contained in the ejaculate. The function of the PSA is to keep the spermatic fluid that coagulates, allowing the sperm to move. PSA is highly specific for prostate tissue, as no other tissue produces it. Free PSA is found free in the plasma, while the bound PSA is complexed to proteins such as alpha-1-antichymotrypsin and alpha-2macroglobulin. The fraction linked to alpha-1-anti -chymotrypsin can be evaluated in serum.

In the early years of the clinical use of free PSA the main international guidelines had adopted a threshold value of $4 \mathrm{ng}$ / $\mathrm{mL}$, then lowered by some authors to $2.5 \mathrm{ng} / \mathrm{mL}$. In fact, for some years it has been widely demonstrated that it is not possible to establish a minimum threshold that can safely rule out the presence of a prostatic carcinoma. A well-known US study [3], conducted on about 3000 men showed a risk of neoplasm of about $25 \%$ for PSA values between 2 and $4 \mathrm{ng} /$ $\mathrm{mL}$ and about $8 \%$ for values less than $1 \mathrm{ng} / \mathrm{mL}$. Therefore, by lowering the PSA reference value there is an increase in the diagnosis of new cases of prostatic neoplasia, but with an increase in the incidence of non-significant tumors, whose natural history does not affect survival. Therefore, the free PSA shows a low diagnostic specificity and is poorly reliable to select clinically significant tumors. For this reason, over the years it has been tried to improve the specificity of PSA with some of its variants such as the relationship between free and total PSA, which finds its rationale in the fact that neoplastic prostate cells produce less PSA in free form than to benign cells. The evaluation of these two fractions of PSA, showed that alterations affecting free PSA are found mainly in benign prostatic hypertrophy, while alterations in the levels of linked PSA are often due to malignant diseases. Therefore, the lower the ratio, the more the risk of cancer increases. For example, when the value falls below $0.10(<0.10)$ the risk of PCa is estimated at $56 \%$. In recent months, among the various PSA isoforms, the -2proPSA has aroused some interest. It is a precursor of free PSA and is typically associated with prostate cancer. The use of this marker significantly improves the diagnostic specificity of the free PSA/total PSA ratio in patients with values of free PSA between 2.5 and $10 \mathrm{ng} / \mathrm{mL}$. In the past almost all doctors considered normal PSA values equal to or less than $4.0 \mathrm{ng} / \mathrm{mL}$, so prostate biopsy for PCa research would be indicated in a subject with values above 4.0 $\mathrm{ng} / \mathrm{mL}$. To complicate the situation, remember that there is no unanimity on the upper limit of normality, for example the National Health Service identifies the value $3.0 \mathrm{ng} / \mathrm{mL}$, the Havard University sets normal limits proportional to age.

\section{Experimental Protocol}

\subsection{Subjects and Treatments}

The patients observed in this survey were recruited after having given their informed consent at AIMI oncological urology clinic. 50 male subjects aged 60 to 75 with a clear diagnosis of $\mathrm{PCa}$, under official cancer therapy, were divided into two groups, the first group (25 patients-Group I) untreated and the second group (25 patients-Group II) treated daily orally with a specific CIZ / PRZ protocol and examined for a period of about four months. During the study the following clinical data collected weekly, were analyzed in all patients,: free and total PSA, PSA density, PHI, PCA3, testosterone, fibrinogen, prostatic acid phosphatase (PAP) and zinc. These clinical parameters were compared with the initial data, placed at 100, obtained before treatment. CIZ and PRZ were obtained from CITOZEATEC, S.r.l. (Peschiera Borromeo, Milano, Italy). The main components of CIZ are as follows (units/100 $\mathrm{g}): 500 \mathrm{mg}$ of vitamin $\mathrm{C} ; 56 \mathrm{mg}$ of vitamin $\mathrm{B} 5 ; 56 \mu \mathrm{g}$ of vitamin D; 3, $3 \mathrm{mg}$ of vitamin B9; $222 \mathrm{mg}$ of pyruvic acid; $120 \mathrm{mg}$ of citric acid; $250 \mathrm{mg}$ of tartaric acid. PRZ is a mixture of glutamic acid $(24.5 \mathrm{mg} / \mathrm{mL})$, acetyl cysteine $(19.3$ $\mathrm{mg} / \mathrm{mL})$, calcium carbonate $(18 \mu \mathrm{g} / \mathrm{mL})$, citric acid $(140$ $\mathrm{mg} / \mathrm{mL})$, tartaric acid $(122.5 \mathrm{mg} / \mathrm{mL})$, sorbitol $(28 \mathrm{mg} / \mathrm{mL})$ and mannitol $(14 \mathrm{mg} / \mathrm{mL})$.

\subsection{Bioassays}

PSA: PSA is still today the most used marker. It is a glycoprotein produced almost exclusively by the epithelial cells of the prostate and, fundamental data, constitutes an organ marker and not of cancer; therefore an elevation of its values can also occur in the presence of other prostatic pathologies (Benign Prostatic Hyperplasia, prostatitis). It is a serine protease of the human tissue callicreine family, encoded by a gene located on chromosome 19 , which is produced by secretory columnar epithelial cells and is contained in the ejaculate. The physiological function of the PSA is to keep the sperm fluid after ejaculation, allowing the spermatozoa to move more easily through the cervix. PSA is highly specific for prostate tissue, as no other tissue produces it. Free PSA is 
found free in the plasma, while the bound PSA is complexed to proteins such as alpha-1-antichymotrypsin and alpha-2-macroglobulin. The fraction linked to alpha-1-antichymotrypsin can be evaluated in serum. In the early years of clinical use of free PSA the main international guidelines had adopted a threshold value of $4 \mathrm{ng} / \mathrm{mL}$, then lowered by some authors to $2.5 \mathrm{ng} / \mathrm{mL}$. In fact, for some years it has been widely demonstrated that it is not possible to establish a minimum threshold that can safely rule out the presence of a prostatic carcinoma. For this reason the free / total PSA ratio is actually considered more reliable from the prognostic point of view. An increase in this value indicates the reduction of tumor progression.

PCA3: PCA3 is a gene overexpressed in $95 \%$ of cases of prostate carcinoma. The value of the messenger RNA (mRNA) of the PCA3 researched in the urinary cells, after prostate massage, is increased $60-100$ times in $95 \%$ of prostate tumors compared to what is observed in normal prostate cells. In addition, its value is independent of the volume of the prostate, inflammation, the rate of blood PSA and the number of biopsies already performed.

PSA density: PSA density expresses the relationship between the total PSA and the measured trans-rectal ultrasound size of the gland, and is based on the fact that the amount of PSA produced per gram of tissue is much greater in cancer than in hypertrophy. PSA density would, in fact, increase the diagnostic accuracy of PSA in patients in whom the marker value is between 4 and $10 \mathrm{ng} / \mathrm{mL}$.

PHI index: the problems arising from the limited ability of the PSA to accurately suggest the presence of a clinically significant prostate tumor allowed to insert a marker called PHI (Prostate Health Index) which derives from a mathematical processing of the data related to three analyzes: total PSA, free PSA and -2proPSA. -2proPSA is a fraction of the PSA molecule that is measured in the blood after a normal sample (p2PSA / PSA free square root PSA total).

Testosterone: a connection between the levels of testosterone and the onset of $\mathrm{PCa}$ has been established, starting from the fact that $\mathrm{PCa}$ cells are initially hormone-sensitive. Low testosterone levels are associated with more aggressive tumors in patients who have developed $\mathrm{PCa}$ [4]. However, this does not mean that having low testosterone levels constitutes a risk or that correcting testosterone levels in those subjects leads to a reduced risk of developing prostate cancer. This association can give us an important indication of how the tumor will evolve before subjecting a patient to surgery and then helping us to choose the best therapy for the individual case. The argument is still controversial, in fact, although testosterone is historically considered an enemy of the prostate, recent clinical and preclinical evidence have reversed this vision. A very large prospective study could clearly demonstrate that there is no association between the concentration of blood testosterone and the risk of prostate cancer [5].

Fibrinogen: globulin that intervenes with other coagulation factors to promote blood coagulation, as it turns into fibrin. Values of 200-400 mg / $100 \mathrm{~mL}$ are considered normal. Values lower than those considered normal can be determined by prostate cancer.

PAP: acid phosphatase is an enzyme that can be measured in the blood, produced by the prostate, spleen, liver, red blood cells, platelets and bone marrow. The dosage of the prostatic fraction serves in particular to confirm or not the suspicion of prostate cancer. They are considered normal values of PAP up to $4.2 \mathrm{mU} / \mathrm{mL}$. Values higher than those considered normal can be determined by prostate hypertrophy and prostate cancer.

Zinc: essential element for normal growth, development of the genital organs, normal prostate activity, wound healing, protein production; controls the activity of more than 100 enzymes and is involved in the functioning of insulin. Small amounts of this element are present in many foods such as lean meat, bread and whole grains, dried beans and marine foods. Normal values: $80-160 \mathrm{mg} / 100 \mathrm{~mL}$.

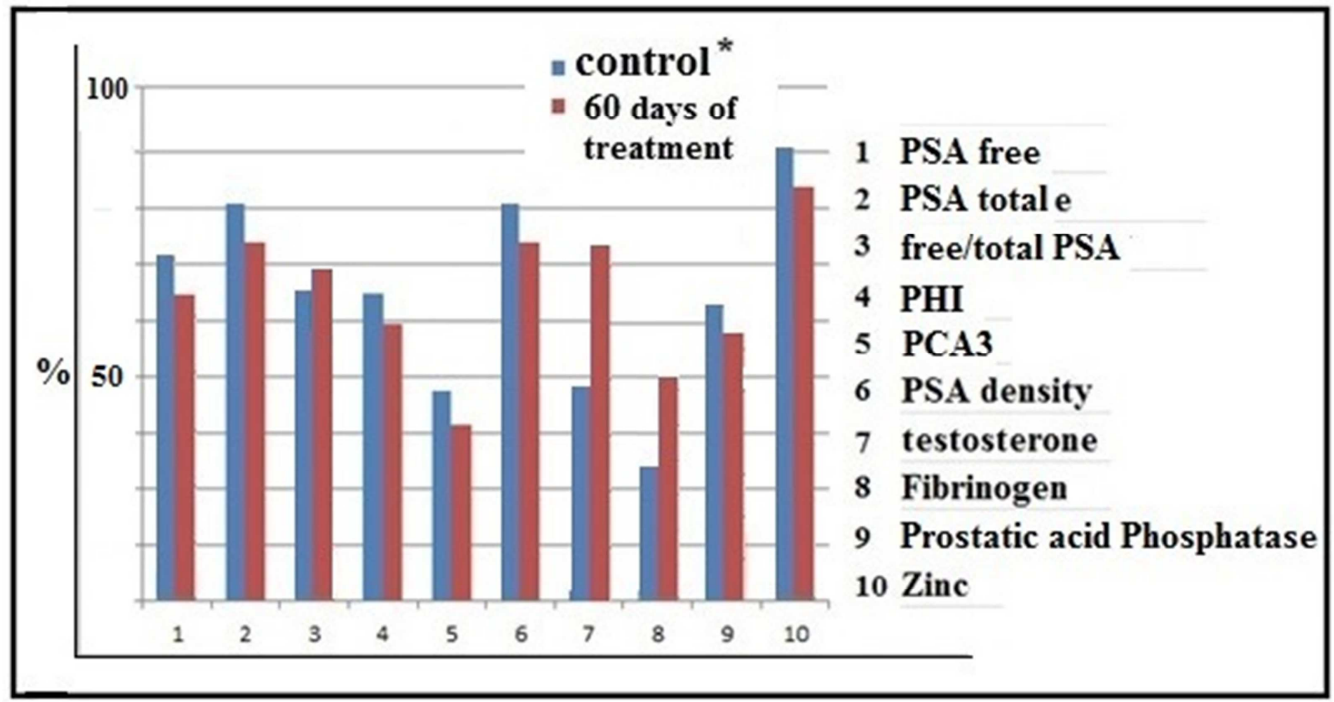

Figure 1. Percentage variation, compared to initial values, of clinical parameters observed in 50 subjects aged between 60 and 75 years with a clear diagnosis of PCa, under official cancer therapy. Patients were divided into two groups. The first group of control (Group I) untreated and the second group (Group II) treated orally for 60 days, with a specific CIZ / PRZ protocol. Individual data showed variations of less than $15 \%$. 


\section{Results}

This study was planned on the basis of a preliminary experimental trial that highlighted the positive activity of CIZ in a model of benign prostatic hypertrophy [6]. Comparing the clinical parameters of patients who have not been treated and treated with CIZ / PRZ for 60, 90 and 120 days, a clear improvement in the effect of the antitumor therapy has emerged. Data processing was performed by comparing the parameters reported in the "Bioassays " section, among the group of patients treated only with the anticancer therapeutic protocol (Group I) and the group of patients treated both with the antitumor protocol and with the two food supplements under examination (Group II). Figure 1 shows the values obtained after 60 days of treatment of the two experimental groups. The adjuvant function exerted on the antitumor therapy, by the two antioxidants CYZ and PRZ, is particularly evident for free and total PSA, whose values are reduced by $7 \%$ and in the ratio free/total PSA increased of about $4 \%$. PCA3 value was reduced of about $7 \%$. The other parameters did not undergo statistically significant changes except for the marked increase in testosterone (30\%) and fibrinogen (17\%) levels. Figure 2 shows the clinical parameters collected after 90 days in patients of Group I and Group II. The reduction of the observed clinical values is already evident only with the antitumor therapy, but becomes even more marked with the aid of antioxidant treatment. In particular, both the free PSA and the total have been reduced by about $20 \%$, improving the value of the free / total PSA ratio. Remarkable is the increase of both the testosterone level $(35 \%)$ and the fibrinogen (27\%). The level of PAP, appears reduced by $13 \%$. Figure 3 shows the values obtained after 120 days of treatment. Both the free PSA and the total PSA are further reduced in Group II, compared to the values of Group I. PHI is reduced by $12 \%$, PCA3 by $10 \%$ and PAP by $15 \%$. Considering the prognostic value of the free / total PSA ratio, the positive result appears the $10 \%$ increase of this parameter following 120 days of treatment.. Values for zinc and PSA density remained unchanged. It is interesting to underline the reduction of testosterone levels, exerted by anticancer therapy, in contrast with the evident total increase of $36 \%$, following the simultaneous treatment with the two antioxidants (CIZ/PRZ).

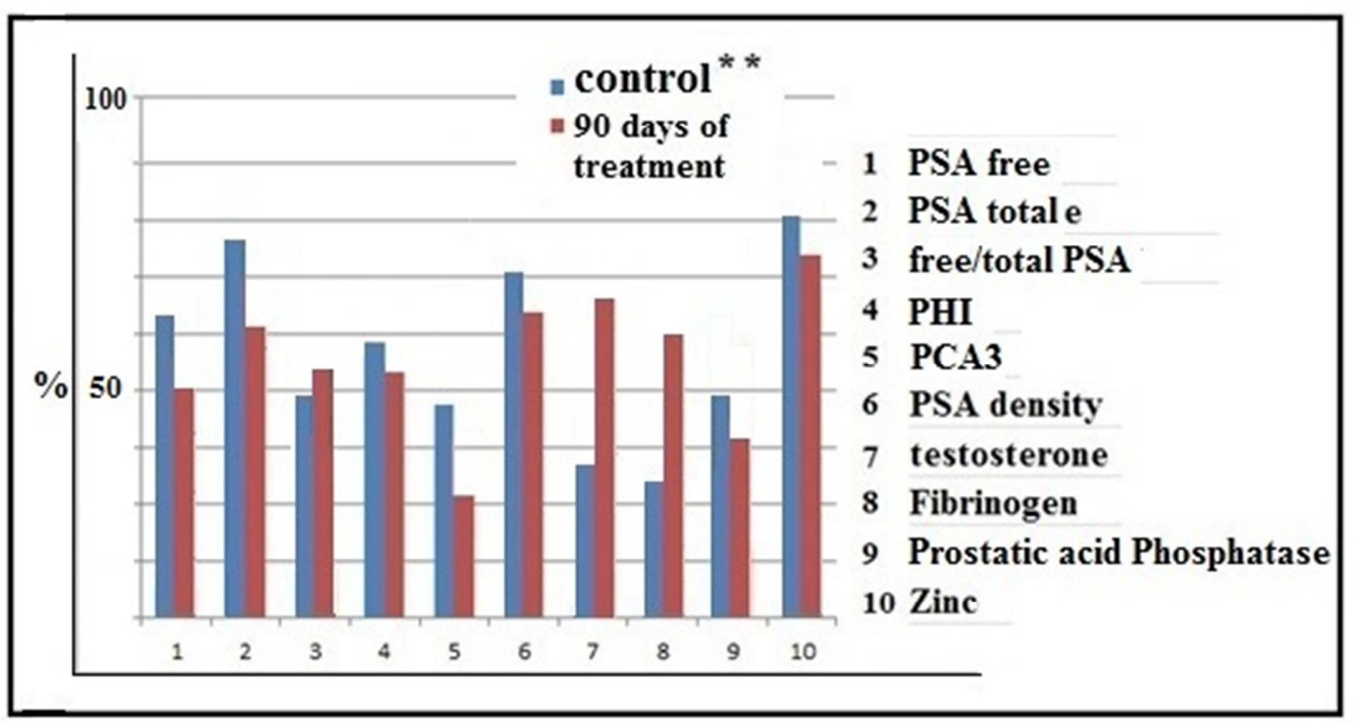

Figure 2. Percentage variation, compared to initial values, of clinical parameters observed in 50 subjects aged between 60 and 75 years with a clear diagnosis of PCa, under official cancer therapy. Patients were divided into two groups. The first group of control (Group I) untreated and the second group (Group II) treated orally for 90 days, with a specific CIZ / PRZ protocol. Individual data showed variations of less than 15\%.

\section{Discussion}

Cancer is one of the major heterogeneous diseases with high morbidity and mortality. Despite extensive research and considerable efforts for developing targeted therapies, it is still an alarming condition with a poor prognosis and high mortality. Numerous studies have provided evidence that changes in redox balance and deregulation of redox signaling are common hallmarks of cancer progression and resistance to treatment. Recent studies have demonstrated that cancer cells are highly adapted to elevated levels of reactive oxygen species (ROS) by activating antioxidant pathways. Thus, targeting the ROS signaling pathways and redox mechanisms involved in cancer development are new potential strategies to prevent cancer.

Based on these considerations, therapy success may be conditioned by the antioxidants present in our own body, which can be synthesized de novo (endogenous) or incorporated through the diet and nutritional supplements (exogenous). Although cells possess a large repertoire of enzymes and antioxidants, sometimes these agents are insufficient to normalize the redox state produced by an intense oxidative stress [7].

In these cases, exogenous antioxidant supplements may be required to restore the cell redox homeostasis [8]. It has been suggested that antioxidant supplementation may protect 
against oxidative stress associated with the development of certain diseases or that it may reverse the oxidative stress produced during their course. In the area of our interest, that is, cancer, antioxidants are acquiring great importance. It is believed that antioxidants can prevent the development of cancer due to their effects on cell cycle regulation, inflammation, the inhibition of tumor cell proliferation and invasiveness, the induction of apoptosis, and the stimulation of the detoxifying enzyme activity [9]. It is interesting to note that it has been reported that daily intake of antioxidants by patients with PCa induces a significant reduction in PSA [1].

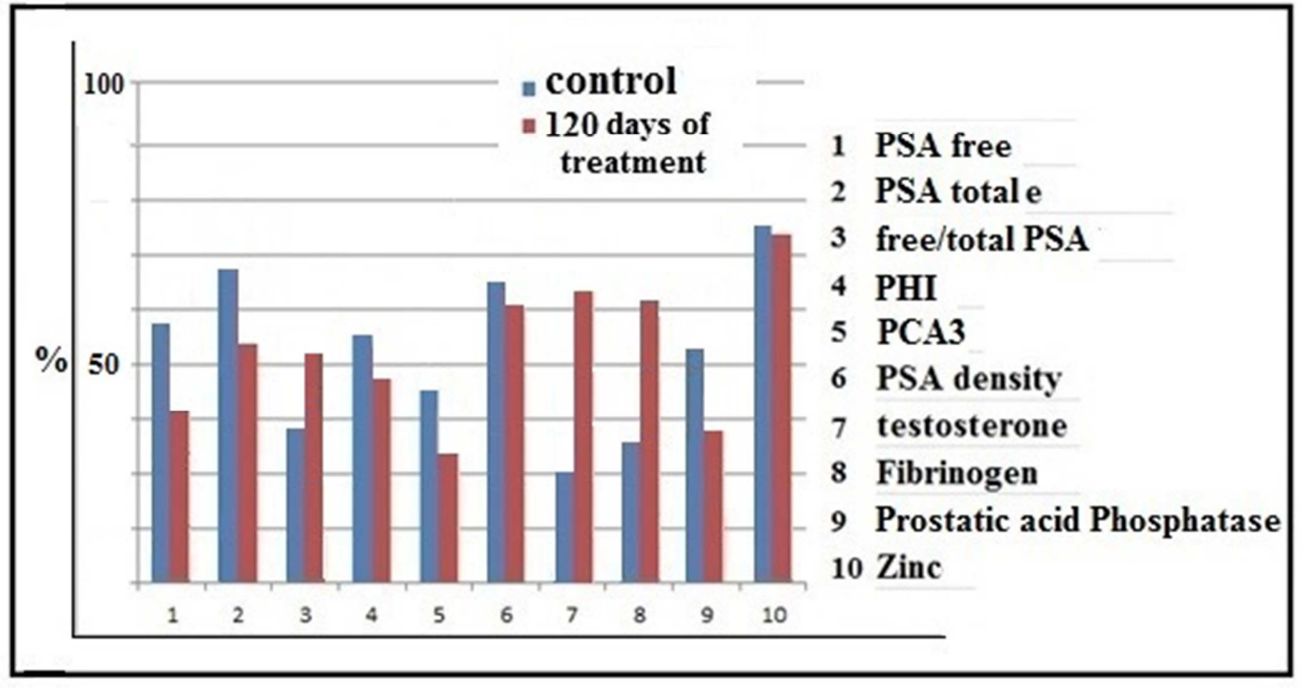

Figure 3. Percentage variation, compared to initial values, of clinical parameters observed in 50 subjects aged between 60 and 75 years with a clear diagnosis of PCa, under official cancer therapy. Patients were divided into two groups. The first group of control (Group I) untreated and the second group (Group II) treated orally for 120 days, with a specific CIZ / PRZ protocol. Individual data showed variations of less than 15\%.

In the present study, two known antioxidant mixtures were administered as adjuvant, during the antitumor therapeutic treatment of patients with PCa. The most interesting data obtained was that of observing a more effective reduction of some biochemical parameters correlated with the evolution of the tumor. In particular, the reduction of free and total PSA observed, has increased the free/total PSA ratio. This data, besides representing a positive observation, could explain the reduction of PHI, of PSA density and of the activity of PAP, as well as the reduction of PCA3. It is also possible to draw from the values obtained, the observation of the improvement of the general condition of the patient (data not shown), thanks to the increase of the levels of testosterone, known protector of the health of the prostate gland [4]. Testosterone is essential for the normal growth, cyto-differentiation and maintenance of prostate tissue [10]. High testosterone levels were previously considered to lead to the potential development of PCa and more rapid growth of the tumor $[11,12]$. However, studies on the association between testosterone and PCa risk, have produced conflicting results $[10,11]$. Numerous studies have demonstrated that low, rather than high, testosterone levels at diagnosis were associated with various markers of poor prognosis, including an advanced pathological stage, higher Gleason scores, higher PSA levels, seminal vesicle invasion and positive surgical margins [13, 14]. Few studies have further investigated whether low levels of testosterone predict poor prognosis. Pending the data on the 120 days of treatment it is already possible a preliminary deduction, that the adjuvant therapy with CYZ and PRZ, appears to improve the biochemical parameters of patients suffering from prostate cancer, probably due to the protective effect of increased testosterone levels. Therefore, further in vivo studies, elucidating the mechanisms underlying the activity of these nutraceuticals, may be useful in the treatment of prostate tumors and reduction of the side effects of chemotherapy. In conclusion the results of the present study may have important clinical implications for the management and treatment of patients with Prostatic Carcinoma.

\section{Acknowledgements}

We are grateful to A. I. A. S. Italy, for financial support for studying the possibility of alternative therapies for prostate cancer (project funding n. SBFAPF00218).

\section{References}

[1] Rahul K. Lall, Deeba N. Syed, Vaqar M. Adhami, Mohammad Imran Khan, and Hasan Mukhtar. Dietary Polyphenols in Prevention and Treatment of Prostate Cancer. Int J Mol Sci. 2015; 16 (2): 3350-3376.

[2] Byeongsang Oh, Gemma Figtree, Daniel Costa, Thomas Eade, George Hruby, Stephanie Lim, Aymen Elfiky, Neil Martine, David Rosenthal, Stephen Clarke, Michael Back. Oxidative stress in prostate cancer patients: A systematic review of case control studies. Prostate Int. 2016; 4: 71-87.

[3] Thompson IM, Pauler DK, Goodman PJ, Tangen CM, Lucia MS, Parnes HL, Minasian LM, Ford LG, Lippman SM, Crawford ED, Crowley JJ, Coltman CA Jr. Prevalence of prostate cancer among men with a prostate-specific antigen level. N Engl J Med. 2004; 350(22): 2239-2246. 
[4] Huakang Tu, Jian Gu, Qing H. Meng, Jeri Kim, Sara Strom, John W. Davis, Yonggang He, Elizabeth A. Wagar, Timothy C. Thompson, Christopher J. Logothetis, and Xifeng Wu. Low serum testosterone is associated with tumor aggressiveness and poor prognosis in prostate cancer. Oncol Lett. 2017 Mar; 13(3): 1949-1957.

[5] Roddam AW, Allen NE, Appleby P, Key TJ. Endogenous sex hormones and prostate cancer: a collaborative analysis of 18 prospective studies. J Natl Cancer Inst. 2008; 100(3):170-183.

[6] Torricelli P., Ferorelli P., De Martino A., Antonelli F., Beninati S. Preventive effects of a mixture of micronutrients with antioxidative properties on experimentally induced prostate hyperplasia American Journal of Life Sciences. 2013; 1(1): 22-26.

[7] Sadowska-Bartosz I, Bartosz G. Effect of antioxidants supplementation on aging and longevity. Biomed Res Int. 2014;2014:404680.

[8] Jaouad Bouayed, Torsten Bohn. Exogenous antioxidants: Double-edged swords in cellular redox state: Health beneficial effects at physiologic doses versus deleterious effects at high doses. Oxid Med Cell Longev. 2010 Jul-Aug; 3(4): 228-237.
[9] Terrence M. Vance, Joseph Su, Elizabeth T. H. Fontham, Sung I Koo, and Ock K. Chun.Dietary Antioxidants and Prostate Cancer: A Review. Nutr Cancer. 2013; 65(6): 217-220.

[10] Isbarn H, Pinthus JH, Marks LS, Montorsi F, Morales A, Morgentaler A, Schulman C. Testosterone and prostate cancer: Revisiting old paradigms. Eur Urol. 2009; 56: 48-56.

[11] Klap J, Schmid M, Loughlin KR. The relationship between total testosterone levels and prostate cancer: A review of the continuing controversy. J Urol. 2015; 193: 403-413.

[12] Morgentaler A. Testosterone and prostate cancer: An historical perspective on a modern myth. Eur Urol. 2006; 50: 935-939.

[13] García-Cruz E, Piqueras M, Huguet J, Peri L, Izquierdo L, Musquera M, Franco A, Alvarez-Vijande R, Ribal MJ, Alcaraz A. Low testosterone levels are related to poor prognosis factors in men with prostate cancer prior to treatment. BJU Int. 2012; 110: E541-E546.

[14] Botto H, Neuzillet Y, Lebret T, Camparo P, Molinie V, Raynaud JP. High incidence of predominant Gleason pattern 4 localized prostate cancer is associated with low serum testosterone. $\mathrm{J}$ Urol. 2011; 186: 1400-1405. 